\title{
Review Article \\ CSF Biomarkers of Alzheimer's Disease: Impact on Disease Concept, Diagnosis, and Clinical Trial Design
}

\author{
Anne M. Fagan \\ Department of Neurology, Knight Alzheimer's Disease Research Center, Hope Center for Neurological Disorders, \\ Washington University School of Medicine, St. Louis, MO 63110, USA \\ Correspondence should be addressed to Anne M. Fagan; fagana@neuro.wustl.edu
}

Received 13 April 2014; Revised 30 June 2014; Accepted 1 July 2014; Published 14 August 2014

Academic Editor: Stephen D. Ginsberg

Copyright (C) 2014 Anne M. Fagan. This is an open access article distributed under the Creative Commons Attribution License, which permits unrestricted use, distribution, and reproduction in any medium, provided the original work is properly cited.

Data from clinicopathologic and biomarker studies have converged to support the view of Alzheimer's disease (AD) as a continuum, with pathology developing decades prior to the onset of cognitive symptoms which culminate as dementia at the end stage of the disease. This concept is impacting disease nomenclature, diagnostic criteria, prognostic potential, and clinical trial design. Revisions to diagnostic criteria to incorporate biomarker results have recently been proposed in order to increase the confidence of $\mathrm{AD}$ as the underlying etiology of a clinical impairment and to permit a diagnosis of $\mathrm{AD}$ across the disease continuum, eventually perhaps in the asymptomatic period. Individuals in this preclinical stage are receiving intense focus as a targeted population for secondary prevention trials aimed at identifying disease-modifying therapies that have the best chance of preserving normal cognitive function. The goal is to bring validated biomarkers to clinical practice for the purpose of disease diagnosis, prognosis, and evaluation of therapeutic efficacy once disease-modifying treatments become available. Realization of this goal requires worldwide biomarker standardization efforts, consensus among researchers and clinicians regarding the clinical utility of assessing biomarkers in patient care settings, and eventually the endorsement and adoption of such procedures and practices into global health care systems.

\section{The Crisis of AD}

Alzheimer's disease (AD) is the most common cause of dementia, accounting for up to $70 \%$ of all dementia cases, and is now estimated to be the third leading cause of death, after heart disease and cancer [1]. Since advanced age is the strongest risk factor for $\mathrm{AD}$, increased life expectancy and the aging of the "baby boomer" generation are leading to dramatic increases in AD incidence. AD currently affects 5.2 million people in the United States (US), with projected estimates reaching 13.8 million (115 million worldwide) by the year 2050 (http://www.alz.org/documents/ national/world_alzheimer_report_2010.pdf). The lifetime risk for $\mathrm{AD}$ dementia for a 65-year-old person is currently estimated to be $\sim 10.5 \%$, with prevalence doubling every 5 years after age 65 , reaching nearly $50 \%$ by age 80 . In the US alone, the costs for care associated with AD in 2013 were more than $\$ 200$ billion, with projected annual costs surpassing $\$ 1$ trillion by the year 2050 (http://www.alz.org/alzheimers_dis- ease_facts_and_figures.asp\#cost). At present there are no effective treatments that will prevent the disease, halt its progression, or delay its onset. Development of an intervention that delays the onset of $\mathrm{AD}$ dementia by 5 years is estimated to result in a $57 \%$ reduction in the number of affected patients and an almost 50\% reduction in projected annual Medicare costs (from $\$ 627$ to $\$ 344$ billion).

Acknowledging the tremendous personal, societal, and financial burdens caused by AD, the National Alzheimer's Project Act (NAPA) was signed into US law (Public Law 111375) in January, 2011, requiring the creation of a national strategic plan to address the rapidly escalating crisis and the coordination of $\mathrm{AD}$ efforts across the federal government. In response, the 2014 US budget allocated $\$ 100$ million specifically for $\mathrm{AD}$ research, education, outreach, and caregiver support. Although certainly helpful, such funding (National Institutes of Health research funds totaling $\sim \$ 560$ million annually for $\mathrm{AD}$ ) still pales in comparison to that allocated to 
cancer, heart disease, and HIV research ( $\$ 3$ billion, $\$ 4$ billion, and $\$ 6$ billion, resp.) (http://www.alz.org/boomers/).

\section{AD Pathology and Diagnosis}

At present, a definitive diagnosis of $\mathrm{AD}$ requires postmortem identification of the two pathologic hallmarks of the disease: extracellular amyloid plaques composed primarily of the aggregated amyloid- $\beta$ (A $\beta)$ peptide and intracellular neurofibrillary tangles composed mainly of the tau protein, a microtubule-associated protein found predominantly in neurons. Quantification and regional distribution of these pathological features have long been implemented in three sets of diagnostic histological criteria (Khachaturian, Consortium to Establish a Registry for Alzheimer Disease (CERAD), and National Institute on Aging-Reagan (NIA-Reagan)) [24], although recent revisions to these criteria have been proposed [5].

Dementia is clinically characterized by a loss or decline in memory and other cognitive abilities from a previously established level of intellectual function that is sufficient to interfere with that person's everyday performance [6]. A clinical diagnosis of $\mathrm{AD}$ is based on criteria established by the National Institute of Neurological and Communicative Disorders and Stroke and the Alzheimer's Disease and Related Disorders Association (NINCDS-ADRDA) [7], although diagnostic sensitivity and specificity are less than desirable, even in specialized memory clinics [8-11]. The 1984 clinical criteria for the diagnosis of probable AD [7] were used in clinical trials and research for almost 30 years. However, during that same time period, knowledge about other (and often combined) causes of dementia such as cerebrovascular disease (stroke), Lewy body disease, and frontotemporal lobar degeneration vastly increased, and pathogenic mutations for dominantly inherited forms of AD were identified [12]. Importantly, $A D$ is now increasingly recognized as a neuropathological entity regardless of clinical status, with the spectrum of the disease ranging from an asymptomatic (preclinical) stage to a prodromal symptomatic stage (mild cognitive impairment or MCI) to the fully expressed symptomatic stage characterized by dementia. This paradigm shift, from considering $\mathrm{AD}$ strictly on the grounds of clinical expression of dementia symptoms to a disease process characterized by a long preclinical period during which pathology is developing and increasing, was facilitated in large part by the development of imaging and fluid (CSF) biomarkers of underlying AD pathologies.

This conceptualization prompted the proposal of two new sets of diagnostic criteria, one from an International Working Group (IWG) [13] and the other from working groups convened by the National Institute on Aging and the Alzheimer's Association (NIA-AA) of the US [14-16]. Although the two sets of criteria differ in some respects, they both aim to support a clinical diagnosis with in vivo evidence of AD pathology (via imaging and/or biofluid biomarkers) and emphasize an etiological diagnosis in the predementia and/or presymptomatic stages of the disease [17] (Table 1). To the extent that such biomarkers are validated and standardized in measurement and interpretation, they will likely eventually be used in clinical practice, especially once a disease-modifying therapy becomes available. As part of that process, biomarkers are being evaluated for their diagnostic and prognostic utility in research cohorts. Such studies have provided critical information regarding the development and progression of $\mathrm{AD}$ pathologic change during the normal course of the disease which, in so doing, is influencing the design and evaluation of current and future clinical trials. The existence of a long presymptomatic period that can be identified by biomarkers has now permitted the initiation of secondary prevention trials of potential disease-modifying therapies in asymptomatic individuals (see below), with the goal of preventing cognitive decline as opposed to halting or slowing progression of symptoms that are already apparent. However, in the absence of effective therapies, it remains to be determined whether a preclinical diagnosis is warranted.

\section{Biomarker Evidence of AD Pathology}

Biomarkers are defined as objective measures of a biological or pathogenic process that can be used to evaluate disease risk or prognosis, to guide clinical diagnosis, or to monitor therapeutic interventions [18]. Both imaging and biofluid biomarkers are being sought and evaluated in AD. Since cerebrospinal fluid (CSF) is in direct contact with the extracellular space of the brain and thus reflects many of the biochemical changes taking place therein, it is considered the optimal source for AD biomarkers. Lumbar puncture (LP) is necessary for the collection of CSF but is perceived as more invasive than a blood draw. The most common adverse side effect of LP is headache, with prevalence of post-LP headache decreasing with increasing age $[19,20]$. Fluid biomarker studies have reported low headache rates $(<2 \%)$ in AD cohorts [21-23] when LPs are performed with "noncutting," atraumatic spinal needles, with few, if any, additional complications. For positional post-LP headaches that do not resolve in a few days with nonprescription pain medications, an epidural blood patch (in which a sample of the person's own blood is injected into the initial LP site) provides immediate relief. Although LPs are typically not included in AD diagnostic evaluations in the US, they are routinely performed for such purposes in many European dementia clinics and centers.

Evaluation of potential CSF biomarkers of $\mathrm{AD}$ began approximately 25 years ago, and many large, longitudinal $\mathrm{AD}$ biomarker studies are currently underway, each testing hypotheses in cohorts defined by specific disease risk profiles, age ranges, and demographics (Table 2). Three proteins that reflect the core neuropathologies are currently considered to be the gold standards of AD CSF biomarkers. These include the 42 amino acid species of $\mathrm{A} \beta(\mathrm{A} \beta 42)$, the primary constituent of amyloid plaques, total tau, and hyperphosphorylated species of tau (ptau) which aggregate to form intraneuronal neurofibrillary tangles.

Concentrations of $\mathrm{A} \beta 42$ in postmortem ventricular CSF [24] and antemortem lumbar CSF $[25,26]$ have been shown to correlate inversely with plaque load at autopsy. This 
TABLE 1: Comparison of new Alzheimer's disease diagnostic criteria proposed by the International Work Group (IWG) and the National Institute on Aging and the Alzheimer's Association (NIA-AA) working groups.

\begin{tabular}{|c|c|c|}
\hline & $\mathrm{IWG}^{1}$ & NIA-AA $^{2}$ \\
\hline $\begin{array}{l}\text { Incorporates biomarkers in the diagnostic } \\
\text { process? }\end{array}$ & Yes & Yes \\
\hline Requires biomarker abnormalities? & Yes & $\begin{array}{l}\text { No-supports diagnosis but is not } \\
\text { required }\end{array}$ \\
\hline $\begin{array}{l}\text { Permits AD diagnosis in the presence of } \\
\text { dementia symptoms? }\end{array}$ & Yes & Yes \\
\hline $\begin{array}{l}\text { Permits AD diagnosis in the absence of } \\
\text { dementia symptoms? }\end{array}$ & $\begin{array}{l}\text { No-"at risk" classification in the } \\
\text { presence of biomarker positivity }\end{array}$ & $\begin{array}{l}\text { Yes-refers to the pathologic process in } \\
\text { the presence or absence of dementia } \\
\text { symptoms }\end{array}$ \\
\hline Considers etiological diagnosis for MCI? & Yes & Yes \\
\hline Classification of MCI & $\begin{array}{l}\text { Replaces the term MCI with "prodromal } \\
\text { AD" (in the presence of biomarker } \\
\text { positivity) }\end{array}$ & Retains the term "MCI" \\
\hline $\begin{array}{l}\text { Required cognitive impairments for MCI } \\
\text { and/or prodromal AD }\end{array}$ & Objective impairment in memory & $\begin{array}{l}\text { Objective or subjective impairment in } \\
\text { memory and/or non-memory domains }\end{array}$ \\
\hline
\end{tabular}

Modified with permission from Morris et al., 2014 [17].

${ }^{1}$ Dubois et al. 2010 [13].

${ }^{2}$ McKhann et al., 2011 [14]; Albert et al., 2011 [15]; Sperling et al., 2011 [16].

TABLE 2: Current CSF biomarker studies of Alzheimer's disease.

\begin{tabular}{|c|c|c|}
\hline Acronym & Study name & Study institution and location \\
\hline ACS & Adult Children Study & Washington University in St. Louis, USA \\
\hline ADNI & Alzheimer's Disease Neuroimaging Initiative & University of Pennsylvania*, USA \\
\hline ARWiBo & Alzheimer's Disease Repository Without Borders ${ }^{\mathrm{mc}}$ & National Alzheimer Centre, Italy \\
\hline \multirow{3}{*}{ API-BIO } & Alzheimer's Prevention Initiative & $\begin{array}{l}\text { University of Antioquia, Colombia, and Banner } \\
\text { Alzheimer's Institute, USA }\end{array}$ \\
\hline & Amsterdam Dementia Cohort ${ }^{\mathrm{mc}}$ & VU University Medical Center, The Netherlands \\
\hline & Arizona APOE Cohort Study & Banner Alzheimer's Institute, USA \\
\hline AIBL & $\begin{array}{l}\text { Australian Imaging, Biomarker and Lifestyle Flagship } \\
\text { Study of Ageing }\end{array}$ & $\begin{array}{l}\text { The Florey Institute of Neuroscience and Mental Health } \\
\text { and Edith Cowan University, Australia }\end{array}$ \\
\hline BIOCARD & Biomarkers for Older Controls At Risk for Dementia & The Johns Hopkins University, USA \\
\hline DESCRIPA & $\begin{array}{l}\text { Development of Screening Guidelines and Criteria for } \\
\text { Predementia Alzheimer's Disease }\end{array}$ & University of Maastricht, The Netherlands \\
\hline DIAN & Dominantly Inherited Alzheimer Network & Washington University in St. Louis*, USA \\
\hline \multirow{2}{*}{$\mathrm{DNC}$} & German Dementia Competence Network ${ }^{\mathrm{mc}}$ & University Erlangen*, Germany \\
\hline & Gothenburg MCI Study ${ }^{\mathrm{mc}}$ & University of Gothenburg, Sweden \\
\hline HASD & Healthy Aging and Senile Dementia & Washington University in St. Louis, USA \\
\hline MCSA & Mayo Clinic Study of Aging & Mayo Clinic, Rochester, USA \\
\hline \multirow[t]{2}{*}{ PLM } & Paris-North, Lille and Montpellier Study ${ }^{\mathrm{mc}}$ & $\begin{array}{l}\text { CMRR: Centres M'emoire de Ressources et de Recherche, } \\
\text { France }\end{array}$ \\
\hline & Unit for Memory Disorders $^{\mathrm{mc}}$ & Lund University, Sweden \\
\hline WRAP & Wisconsin Registry for Alzheimer's Prevention & University of Wisconsin, Madison, USA \\
\hline
\end{tabular}

${ }^{*}$ Coordinating Center.

${ }^{\mathrm{mc}}$ Memory clinic.

relationship is also observed in living individuals, with low concentrations of CSF $\mathrm{A} \beta 42$ associated with the presence of plaques as determined by in vivo amyloid imaging via positron emission tomography (PET) [27-31]. Such findings support the concept that levels of CSF A $\beta 42$ are reduced in $\mathrm{AD}$ due to its sequestration in parenchymal plaques which limits its ability to diffuse into the CSF [32]. However, the relationship is not perfect since some individuals exhibit low levels of CSF A $\beta 42$ in the absence of appreciable cortical amyloid load by imaging [33]. Whether this inconsistency reflects limitations of either or both of the two biomarker methodologies or is indicative of true biology remains to 
be determined. Each amyloid biomarker modality has its own strengths and weaknesses. There are currently three amyloid PET tracers approved for clinical use by the United States Food and Drug Administration (FDA) (florbetapir (Amyvid), Eli Lilly and Company; flutemetamol (Vizamyl), GE Healthcare; florbetaben (Neuraceq), Piramal Imaging). While a PET scan is viewed by many as less invasive than lumbar puncture, it is much more costly and poses additional risks due to injection of a radioactive tracer. However, unlike CSF, amyloid imaging is able to provide information regarding regional patterns of pathology. In comparison, CSF is able to provide information regarding multiple $\mathrm{AD}$ related biomarkers (e.g., amyloid, tangles, neuroinflammation, neuronal injury/death, and other processes yet to be determined), albeit without regional specificity. Although AD CSF biomarkers have not yet been approved by the FDA, clinical samples are routinely assessed for diagnostic purposes in several European countries as well as the US, albeit much less frequently than in Europe. Efforts to obtain FDA approval for the use of CSF biomarkers in US clinical settings are currently underway.

Tau is the primary component of neurofibrillary tangles in $\mathrm{AD}$, but tau aggregates are the defining features of several other tauopathies including frontotemporal dementia (FTD), progressive supranuclear palsy (PSP), chronic traumatic encephalopathy (also known as dementia pugilistica), corticobasal degeneration, and Pick's disease. However, results from studies evaluating CSF levels of tau in the various tauopathies have been mixed likely due to the large heterogeneity among these clinical syndromes [34]. In contrast, CSF tau is consistently reported to be elevated in conditions involving robust neuronal cell death, including acute stroke $[35,36]$, traumatic brain injury (TBI) [36, 37], CreutzfeldtJakob disease (CJD) [38-40], and $\mathrm{AD}$ [18], and thus is considered to be a marker of neuronal injury and/or death. However, both soluble and aggregated forms of tau have been shown to be secreted from healthy cells in culture [41], and evidence from animal studies suggests tau is continuously secreted from healthy neurons into the brain interstitial fluid space [42], perhaps driven by synaptic activity [43].

Although levels of ptau are typically highly correlated with levels of tau in the CSF, ptau is considered to be a more specific biomarker for $\mathrm{AD}$ since elevations in ptau are not observed following traumatic brain injury and stroke or in CJD [44]. Instead, ptau is likely more a marker of neurofibrillary tangles since positive correlations have been observed between levels of ptau ( $\mathrm{p}-\mathrm{tau}_{181}$ and $\mathrm{p}-\mathrm{tau}_{231}$ and tau phosphorylated at residues 181 and 231, resp.) and neocortical tangle pathology at autopsy [25, 45]. Future studies evaluating the association between tangle load via tau PET imaging [46] and CSF levels of tau and ptau will be very informative in defining the pathological etiology of elevated CSF tau and ptau. Curiously, levels of ptau in other neurodegenerative conditions known to have tangle pathology, while typically elevated in comparison to nondiseased controls, do not reach the levels observed in AD [34]. Whether this apparent discrepancy reflects the inherent heterogeneity in the many tauopathies (with differences in tangle load and/or regional distributions and its pathological course) that impacts the ability of ptau to reach the CSF, potentially different phosphorylation states in these other tauopathies that are not detected by the current ptau assays, or other factors remains to be determined.

Unbiased proteomics screens and targeted multianalyte surveys of CSF have identified many proteins that are elevated or reduced in $\mathrm{AD}$ compared to cognitively normal controls [47]. Many candidate proteins are presumed markers of oxidative stress, synapse loss or neurodegeneration, and neuroinflammation, processes known to be involved in $\mathrm{AD}$, albeit nonspecifically. It is likely that these markers will be more useful in disease staging than for disease diagnosis. For example, visinin-like protein 1 (VILIP-1) and YKL-40 (also known as chitinase-3-like protein 1), presumed markers of neurodegeneration and neuroinflammation, respectively, have been shown to be elevated in AD CSF compared to controls [48-53], although with overlap between the clinical groups. However, these markers, in the presence of low levels of CSF $\mathrm{A} \beta 42$, are strong predictors of future cognitive decline/dementia (within 3-4 years) in elders who are cognitively normal or who have MCI/very mild AD [49-54], suggesting they are able to identify a stage of $\mathrm{AD}$ just prior to symptomatic expression. Despite such promise, until these candidate markers are validated in additional large research cohorts, the three core biomarkers remain the gold standards.

\section{Diagnostic and Prognostic Performance of CSF Biomarkers}

Biomarker sensitivity and specificity are both important for $\mathrm{AD}$ diagnosis. A high sensitivity is required to identify the highest proportion of individuals with $\mathrm{AD}$ and minimize false negatives, whereas a high specificity is necessary for diagnostic accuracy to minimize false positives and exclude symptomatic or prodromal cases due to non-AD pathological processes. However, the criteria for what is considered "high" or even "acceptable" will likely differ depending on the purpose for which the biomarkers will be used, such as providing a disease diagnosis, primary care screening for referral to specialized memory clinics, clinical trial enrollment, or eventual treatment with disease-modifying drugs that may be expensive and/or have unwanted side effects. The high prevalence of mixed pathologies in individuals with $\mathrm{AD}$ will also likely impact biomarker criteria. All of these considerations are topics of ongoing investigation.

4.1. AD Dementia. Since the current criteria for the clinical diagnosis of $\mathrm{AD}$ require the presence of dementia [7], the majority of biomarker studies over the years have evaluated the ability of candidate markers to distinguish individuals clinically diagnosed with $\mathrm{AD}$ from those without dementia. In such analyses, elevations in CSF tau and ptau ( 300\%) and reductions in $\mathrm{A} \beta 42(\sim 50 \%)$ are observed in $\mathrm{AD}$, typically with sensitivities and specificities greater than $80 \%$ [55]. This biomarker accuracy, while good, is not better than current clinical accuracies, at least in specialized dementia centers and clinics [8]. This suboptimal accuracy is likely due to the fact that $25-30 \%$ of cognitively normal elders are known to 
have enough plaques and tangles to warrant a neuropathological diagnosis of AD (presumed "preclinical" AD) [56, 57], thus "contaminating" the cognitively normal group with individuals with underlying AD pathology. Despite this caveat, a combined measure of these biomarkers (e.g., high levels of tau(s) and low levels of A $\beta 42$ ) performs better than the single biomarkers on their own $[58,59]$. Importantly, these markers are normal in several other disorders which are often accompanied by cognitive impairment such as depression and Parkinson's disease (PD) [37]. In addition, CSF ptau, in particular, can aid in the differentiation of $\mathrm{AD}$ from other dementias, such as FTD and Lewy body dementia (DLB) [44]. However, the overall diagnostic performance of these CSF biomarkers to discriminate AD dementia from dementia due to other etiologies is not optimal, likely reflecting the relative abundance of mixed pathologies in people presenting with dementia $[60,61]$, as well as inaccuracies in differential clinical diagnosis, especially at early disease stages. Combined with the presence of preclinical pathology in a substantial percentage of nondemented elderly individuals, assessing biomarkers based on clinical diagnoses precludes the possibility of finding a disease biomarker with 100\% sensitivity and specificity for AD. Nonetheless, proposals have been made to include biomarker profiles in diagnostic formulations for probable and possible $\mathrm{AD}$ dementia for use in research and clinical settings [13, 14].

4.2. Prodromal AD/Mild Cognitive Impairment. Longitudinal study of older individuals over time has been critical for our understanding of $\mathrm{AD}$ as a disease continuum. Furthermore, given the consistently poor results in $\mathrm{AD}$ clinical trials to date which have enrolled individuals who already have dementia, it has been proposed that development of disease-modifying drugs will likely require testing in patient populations at very early (predementia) stages. This critical interest in early diagnosis, coupled with the appreciation of the long time period during which $\mathrm{AD}$ pathology develops prior to the symptoms of dementia (see below), has fueled efforts to identify biomarkers of AD pathology at earlier disease stages. Mild cognitive impairment (MCI) has been recognized as a possible intermediate stage between normal aging and $\mathrm{AD}$ dementia $[62,63]$ and, as such, has been the subject of intense interest in the $\mathrm{AD}$ biomarker field. As a group, individuals with MCI (defined by impairments in cognitive abilities compared to age-matched normative values but at levels that are below the threshold considered to be "dementia") typically display mean $\mathrm{AD}$ biomarker profiles of CSF $\mathrm{A} \beta 42$, tau, and ptau intermediate to those with $\mathrm{AD}$ dementia and nondemented controls $[55,64]$. However, there is significant overlap among individuals in the MCI and the other diagnostic groups, with approximately twothirds of individuals with MCI displaying profiles consistent with $\mathrm{AD}$ pathology and one-third appearing in the normal range [65]. Such heterogeneity likely reflects differences in the underlying etiologies of the cognitive impairments. Indeed, amyloid plaques, as visualized by PET imaging, are present in $\sim 60 \%$ of individuals with MCI [66], and longitudinal studies in several international cohorts have demonstrated that the combination of low CSF A $\beta 42$ and high CSF tau and ptau is highly predictive of which MCI cases will progress to $\mathrm{AD}$ dementia (i.e., prodromal $\mathrm{AD}$ ) [65, 67-69], as well as their rate of cognitive decline [54, 70]. Such studies have prompted the proposed use of these CSF biomarkers to define (probabilistically) the underlying etiology of MCI cases in research as well as clinical settings [15].

4.3. Asymptomatic/Preclinical AD. Prevention of cognitive decline (prior to irrevocable neuronal cell loss) is the ultimate goal of $\mathrm{AD}$ therapeutics. However, implementation of such a strategy provides a unique challenge since, by definition, clinical measures will be unable to identify individuals in this presymptomatic phase. Therefore, the use of validated disease biomarkers will be required for such patient identification. Data from clinicopathological and biomarker studies have converged to support the existence of a long preclinical period stage (estimated to be at least 10-20 years) during which the pathologies of $\mathrm{AD}$ develop before the appearance of any cognitive symptoms of dementia. Roughly one-third of cognitively normal elderly individuals who come to autopsy meet the histopathological criteria for $\operatorname{AD}[56,71,72]$. Data from more recent biomarker studies in large cohorts of cognitively normal individuals confirm this finding; a similar proportion exhibits low levels of CSF A $\beta 42$ that are indicative of the presence of cortical amyloid as detected by PET amyloid imaging [27-31]. While some asymptomatic individuals exhibit high levels of CSF tau in the absence of amyloid positivity (defined by CSF A $\beta 42$ ) [73], the etiology(ies) of such elevations and their impact on clinical performance are not clear.

The critical element required for establishing biomarker validity and utility in the preclinical period is its ability to predict future dementia. Low levels of CSF A $\beta 42$ have been shown to predict future cognitive decline and/or AD dementia in cognitively normal elders [75-77]. In those same studies, high levels of tau and ptau were not useful predictors of cognitive decline. However, more recent studies have shown that the combination of these CSF pathologic markers (e.g., low $\mathrm{A} \beta 42$ and high tau(s) or a high tau(s)/A $\beta 42$ ratio) in cognitively normal elders is highly predictive of future cognitive decline (within a few years) $[49,50,78,79]$, similar to its ability to predict cognitive decline and/or AD dementia in MCI cohorts [70,80]. Visinin-like protein 1, a neuronal calcium sensor protein that is currently receiving a lot of interest as a novel, tau-independent marker of neurodegeneration, is elevated in $\mathrm{AD}[48,53]$ and has similarly been shown to be a strong predictor of cognitive decline and/or dementia in cognitively normal elders in the presence of low levels of A $\beta 42[50,54]$.

Arguably the most compelling support for the utility of biomarkers in predicting cognitive decline and dementia comes from studies of rare families with known AD-causing mutations (autosomal-dominant AD (ADAD) that comprises $\sim 1 \%$ of all $\mathrm{AD}$ cases). These individuals have been excluded from observational studies and clinical trials due to the genetic mechanism of their disease. However, despite potential differences in the mechanisms by which $\mathrm{A} \beta$ accumulates 
to form amyloid in the brain (likely due to the increase in A $\beta 1$ 42 production in ADAD compared to more complex and still poorly understood mechanism(s) in late-onset AD (LOAD) [81]), the hallmark pathologies and clinical symptoms are similar between the two disease groups [82]. Study of ADAD is particularly well-suited to investigation of biomarker utility, especially during the preclinical/asymptomatic period, since the $100 \%$ penetrance of the mutations and relative consistency of age at symptom onset within families allows investigators to know with certainty who will develop dementia and the approximate timing of symptom appearance. These characteristics overcome the limitations of disease uncertainty and unpredictability of symptom onset inherent in studies of LOAD. Symptomatic mutation carriers exhibit low levels of CSF A $\beta 42$ compared to mutation noncarriers [83-85] (as do individuals with Down syndrome who are destined to develop $\mathrm{AD}$ due to the presence of three copies of the gene for the amyloid precursor protein, $A P P$; [86]) and high levels of tau $[84,85,87]$. Furthermore, recent reports from the Dominantly Inherited Alzheimer Network (DIAN) indicate that these $\mathrm{AD}$ biomarker signatures are observed at least $\sim 10$ to 20 years prior to carriers' predicted age of symptom onset (defined as the age at which their parent developed clinical symptoms) $[84,85]$. The precise timing of such preclinical changes in the more common form of the disease (LOAD) and when they are first detectable are topics of current investigation and will be critical to consider if an $\mathrm{AD}$ diagnosis is to ever be made in the absence of cognitive symptoms [16].

\section{Biomarker Use in Tracking Disease Progression}

Over the past decade great progress has been made in the AD biomarker field in identifying markers that not only yield a more accurate disease diagnosis and prognosis in research cohorts, but also provide insight into the normal trajectories of neuropathologic changes over the course of the disease, especially during the early and preclinical stages [88]. Results from many cross-sectional studies support a model in which dynamic reductions in CSF A $\beta 42$ (indicative of amyloid plaques) occur in the early and preclinical phase, with levels remaining fairly constant during the subsequent symptomatic phase, followed by elevations in markers of neuronal injury (such as tau and ptau and, more recently, VILIP-1) later in the disease and that continue as people approach and move through the symptomatic phase $[89,90]$ (Figure 1(a)). Consistent with this model, cognitive decline is more closely related to markers of neuronal injury than amyloid load [91-93].

Recent preliminary findings from within-person longitudinal biomarker analyses in DIAN support inclusion of an eventual slowing down of the rate of neuronal injury with symptomatic disease progression. While still cognitively normal, mutation carriers exhibit increases in CSF tau (and $\operatorname{ptau}_{181}$ and VILIP-1), consistent with results of crosssectional analyses $[84,85]$. However, these same markers of neuronal injury actually decrease in mutation carriers who are symptomatic [85]. Although relatively small in number, studies investigating within-person change in LOAD have demonstrated little or no change in biomarker levels over relatively short time periods (6 months to 2 years) [9497]. A closer review of the data, however, reveals betweensubject variability in the patterns of tau changes that may be biologically relevant. For example, tau was observed to increase in persons with LOAD who had low tau at baseline (presumably early in the course of the disease) but showed no difference or even decreases in those with high baseline levels (presumed to be later in the disease process) [98, 99]. Consistent with this finding, statistical modeling in the Alzheimer's Disease Neuroimaging Initiative (ADNI) cohort revealed similar reductions in tau in $\mathrm{AD}$ cases but not in those with MCI [100]. Longitudinal biomarker studies of middleaged individuals who are followed from cognitive normality through mild cognitive impairment to AD dementia will provide critical information regarding biomarker changes over the entire course of the disease, from preclinical to $\mathrm{MCI} /$ prodromal $\mathrm{AD}$ to $\mathrm{AD}$ dementia. Such information will be important for patient care in the long run and currently for informing the design and interpretation of clinical trials of disease-modifying therapies.

\section{The Potential Use of Biomarkers in Clinical Practice}

Although measurements of CSF A $\beta 42$, tau, and ptau in clinic patients can already be obtained by physicians in the US and in many European countries, these measures are not part of the clinical criteria for the diagnosis of AD dementia. However, this may soon change. The current clinical diagnostic criteria for AD were developed over 25 years ago by the NINCDS-ADRDA Work Group [7]. These criteria depend largely on the exclusion of causes other than $\mathrm{AD}$ for dementia and state that a diagnosis of $\mathrm{AD}$ cannot be made until the patient has dementia, defined as "cognitive symptoms severe enough to interfere with social or occupational activities" [7]. The Diagnostic and Statistical Manual fourth revision (DSMIV) and International Classification of Disease tenth revision (ICD-10) criteria, which are used for routine diagnosis, also require that a patient demonstrate dementia before a diagnosis of $\mathrm{AD}$ is possible. Given that we now know that dementia is the end stage of a long disease process and predict that disease-modifying therapies will likely be most beneficial if administered earlier in the disease course, new criteria for different stages of $\mathrm{AD}$ across the disease continuum have recently been proposed by three work groups convened by the NIA-AA [14-16]. Similar (but not identical) revisions have also been proposed by the IWG $[13,17]$. These new criteria differ from the ones published in 1984 in the fact that they incorporate biomarkers of the underlying disease state and use them to formalize the different disease stages [101]. These criteria have been developed, in principal, to increase the confidence of $\mathrm{AD}$ as the underlying etiology of a clinical impairment and permit a diagnosis of $\mathrm{AD}$ at earlier disease stages, even before the emergence of symptoms. Efforts to 

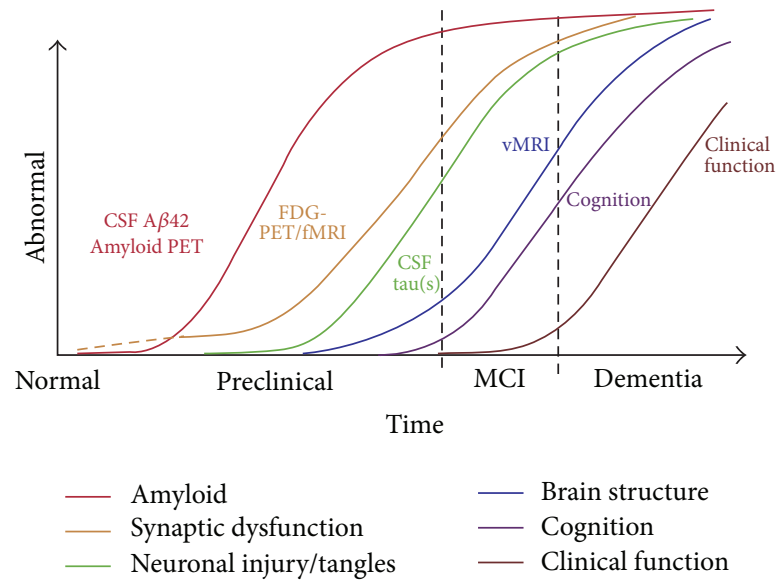

(a)

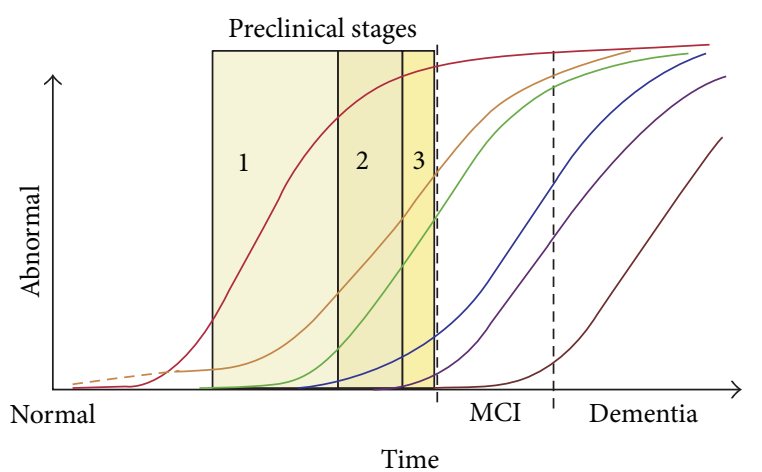

$\begin{array}{ll}- \text { Amyloid } & - \text { Brain structure } \\ - \text { Synaptic dysfunction } & - \text { Cognition } \\ - \text { Neuronal injury/tangles } & - \text { Clinical function }\end{array}$

(b)

FIGURE 1: Hypothesized model of biomarker trajectories in AD based on cross-sectional data. (a) Amyloid (red) is identified by CSF A $\beta 42$ or amyloid positron emission tomography (PET). Synaptic dysfunction (gold) is evidenced by fluorodeoxyglucose [F18] PET (FDG-PET), with dashed line indicating that abnormalities may be detected earlier than amyloid or functional magnetic resonance imaging (fMRI). Neuronal injury and/or neurofibrillary tangles are detected by CSF tau (and phosphorylated tau) (green), and brain structure/volume is evaluated by volumetric MRI (vMRI) (blue). Cognition is evaluated by clinical and psychometric testing (purple), whereas clinical function refers to cognitive and behavioral abilities that impact daily living (brown). Biomarkers change from normal to maximally abnormal ( $Y$-axis) as a function of time ( $X$-axis), with typical clinical disease stages (preclinical, MCI, and dementia) separated by vertical dashed lines. (b) The long preclinical stage is further characterized by hypothesized progressive stages. Stage 1 is defined as asymptomatic cerebral amyloidosis (abnormal CSF A $\beta 42$ and/or amyloid PET). Stage 2 is defined as asymptomatic cerebral amyloidosis with concomitant evidence of neuronal injury/degeneration (abnormal CSF tau/ptau and/or vMRI). Stage 3 is defined as asymptomatic cerebral amyloidosis with evidence of neuronal injury/degeneration and subtle cognitive/behavioral decline (as defined by clinical and/or psychometric testing). Adapted with permission from Jack et al. [90].

define the perceived and practical utility of biomarker data in clinical settings are ongoing [102-104].

6.1. AD Dementia. The revised NIA-AA criteria for defining $\mathrm{AD}$ dementia expand the breadth of the 1984 criteria for the clinical diagnosis of probable $\mathrm{AD}$ dementia and better define the criteria for possible $\mathrm{AD}$. In addition, for use in research settings, biomarkers are integrated into the diagnostic formulations for both of these designations [14] (Table 3).

6.2. $M C I$ due to $A D$. The NIA-AA criteria to identify the symptomatic but predementia phase of $\mathrm{AD}$ (MCI due to AD) refine previous clinical definitions of MCI and add an entity of $\mathrm{MCI}$ due to $\mathrm{AD}$ pathology based on the combination of clinical diagnosis and the presence of $\mathrm{AD}$ biomarkers, resulting in four levels of diagnostic certainty [15] (Table 3).

6.3. Preclinical $A D$. By definition, the NIA-AA criteria for preclinical $\mathrm{AD}$ (currently for research use only) are based almost entirely on biomarkers. The goal of these criteria is to define the factors in cognitively normal individuals that best predict the emergence of clinical impairment and progression to eventual $\mathrm{AD}$ dementia so to be able to identify persons most likely to ultimately benefit from early therapeutic intervention [16]. These criteria proposed ordered stages for cognitively normal individuals with abnormal amyloid markers (stage 1), abnormal amyloid and injury markers (stage 2), and abnormal amyloid and injury markers and subtle cognitive changes (stage 3) (Table 3, Figure 1(b)). A subsequent study using imaging variables to operationally define the preclinical stages proposed the addition of stage 0 to identify people without disease (defined by cognitive normality and biomarker negativity), as well as a classification that identifies people with Suspected Non-Alzheimer Pathophysiology (SNAP) (defined by positive injury markers in the absence of amyloid) [74] (Table 3). In support of the utility of these stages for predicting the timing of future cognitive impairment in cognitively normal individuals, short-term (1 year) progression rates to $\mathrm{MCI}$ or dementia were reported to increase with advancing preclinical AD stage when structural and amyloid imaging measures were used for staging [105]. Consistent with this finding, a recent study using CSF biomarkers as staging measures reported that individuals with preclinical $\mathrm{AD}$ (cognitively normal with positive $\mathrm{AD}$ biomarkers) exhibited a faster rate of progression to MCI and/or $\mathrm{AD}$ dementia compared to biomarker-negative individuals, with progression rates differing among the preclinical AD stages (stage 3 faster than stage 2; stage 2 faster than stage 1) [73]. Furthermore, the presence of preclinical AD as defined by these criteria was also associated with a higher mortality risk, which also increased with advancing stage [73]. Together, these data demonstrate the prognostic utility of the proposed NIA-AA stages for preclinical AD for the prediction of future cognitive decline and mortality in cognitively normal elders and support the potential use of 


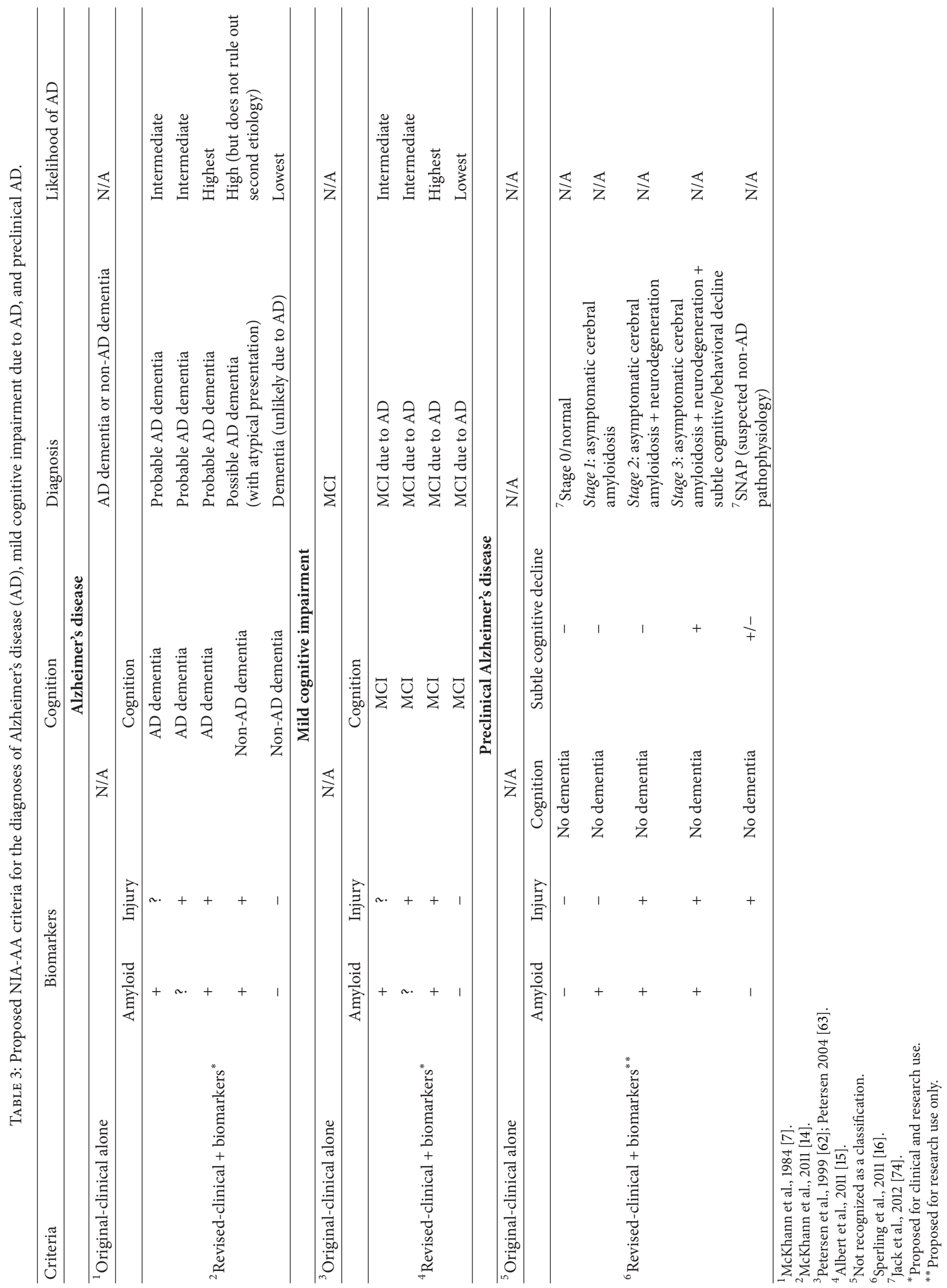


NIA-AA criteria in research studies as well as in AD clinical trials. Whether such criteria will eventually be considered in primary care settings remains to be determined and will most likely depend on the availability of effective diseasemodifying therapies.

\section{Use of Biomarkers in Clinical Trials}

To date all clinical trials of putative disease-modifying therapies in individuals with $\mathrm{AD}$ dementia have failed to show meaningful clinical benefit. Such failures may be due to several factors including inadequacies of the specific drugs tested, inappropriate pathobiological targets, inclusion of misdiagnosed patients, use of insensitive cognitive outcome measures, and/or enrollment of individuals who are too far progressed in the disease (i.e., demented) and thus have already undergone substantial neuronal cell death [106], in other words, perhaps the wrong drug, the wrong target, the wrong people, and/or the wrong stage [107].

To address these potential shortcomings, biomarkers are being used in current and planned AD secondary prevention trials of potential disease-modifying therapies [108]: (1) Anti-Amyloid Treatment in Asymptomatic Alzheimer's Disease (A4 Study) (http://www.clinicaltrials.gov/ct2/show/ NCT02008357?term=A4\&rank=1) [109]; (2) Alzheimer's Prevention Initiative (API) (http://www.clinicaltrials.gov/ ct2/show/NCT01998841?term=alzheimer\%27s+prevention+ initiative\&rank=1) [110]; and (3) Dominantly Inherited Alzheimer Network Trial Unit (DIAN TU) (http://www.clinicaltrials.gov/ct2/show/NCT01760005?term=DIAN\&rank=2) [111]. For example, levels of CSF A $\beta 42$, tau, and/or ptau (and/or imaging measures) are being used to (1) enrich the number of patients who actually have underlying AD pathology; (2) stratify patients according to the presence and/or the amount of underlying pathology; (3) assess therapeutic target engagement; and (4) monitor the effects of treatment on downstream pathogenic processes. Not only will this approach result in an overall reduction in required patient numbers, trial duration, and cost, but it is also expected to provide information particularly suitable for making go/no-go decisions as to whether to move candidate therapies forward into large and expensive Phase II and III trials. There is also growing interest in the role biomarkers could play as surrogate endpoints in treatment trials, such that treatment effects on the biomarkers themselves could be used as a replacement for clinical endpoints in the accelerated approval of $\mathrm{AD}$ therapies by regulatory agencies $[18,106]$.

\section{Challenges and Opportunities}

8.1. Biomarker. The AD biomarker field is faced with several challenges that must be overcome in order to move promising analytes into clinical practice. From a methodological perspective, biomarker candidates must be validated in large, well-characterized research cohorts, with care taken to evaluate the potential impact of important $\mathrm{AD}$-related covariates such as age, gender, and $A P O E$ genotype (the strongest genetic risk factor for $\mathrm{AD}$ ). The field is close to achieving that. However, protocol and assay standardization must still be attained in order to maximize biomarker sensitivity and reliability. Although biological and within-laboratory variability for CSF markers are low, there is variation in biomarker levels between laboratories. Furthermore, there are no available calibrators (certified reference standards) for these biomarkers and no consensus on what assays should be used [18]. Worldwide biomarker standardization efforts are currently underway [112-114], and in pharmaceutical and biotech laboratories, emphasis is being placed on development of commercial assay platforms with automated, highthroughput capabilities.

Given the long preclinical stage of $\mathrm{AD}$, biomarker validation should define sensitivity and specificity (and associated positive and negative predictive values) for underlying $\mathrm{AD}$ pathology, not merely clinical diagnosis that is often inaccurate. To date, very few studies have been able to correlate biomarkers in antemortem samples with results of histologic postmortem examination, and the time interval between the two evaluations can be large. As in vivo amyloid PET imaging provided the opportunity to validate CSF A $\beta 42$ as a marker of amyloid, methods to image neurofibrillary tangles $[46,115]$ and neuroinflammatory responses [116] are in development and will be very informative for providing pathologic validation for CSF tau and ptau and potential markers of neuroinflammation such as YKL-40. Establishing valid cut-points to define biomarker positivity versus negativity [117] will ultimately be required in order to eventually bring biomarkers to the clinic. Such cut-points will likely also depend on covariates such as age, genetic risk factors (e.g., APOE genotype), and or family history of $\mathrm{AD}$. In addition, studies evaluating the change in biomarker profiles within individuals with disease progression will be important for disease staging purposes, as will be the development of statistical methods to determine cut-points for such changes over time [118]. Evaluation of biomarker utility for differential diagnosis in cohorts with known underlying nonAD pathologies (FTLD, DLB, vascular dementia, etc.) is also needed, especially since misdiagnoses often occur at early clinical stages. It is likely that combinations of biomarkers will prove most useful for disease diagnosis (presence versus absence of $\mathrm{AD}$ pathology) and prognosis (predicting cognitive decline), either within modalities or between modalities (e.g., fluids and imaging) $[89,119,120]$. Ongoing studies that collect multiple types of biomarker data will be critical for such assessments. Finally, although considered to be the Holy Grail, it remains to be determined whether any viable plasma biomarkers will emerge. Many research groups are actively engaged in the search given the clinical appeal of diagnostic blood tests [121].

8.2. Clinical. Lumbar puncture is considered to be more invasive than blood draw, and negative attitudes towards LP provide a challenge to implementing AD biomarkers in both research and clinical settings. The most prevalent adverse side effects of LPs are mild-moderate headache and backache, with more severe headaches considered to be the primary safety concern. However, use of small caliber "noncutting" 
atraumatic spinal needles (e.g., Sprotte or Whitacre 22-25 gauge) has been shown to significantly reduce the incidence of post-LP headaches $[21,23,122]$ such that their use is now recommended by experts in the field [123].

On the clinical front, ethical, policy, and social challenges exist regarding provision of a preclinical diagnosis, especially in the absence of an available disease-modifying therapy. Challenges for the physician include understanding if and when a cognitively normal patient should undergo testing and the potential harm (e.g., anxiety, depression) a patient may experience upon receiving a diagnosis of preclinical $\mathrm{AD}$ [124]. Challenges also exist regarding the translation of diagnosis of preclinical AD into practice and policy. Privacy and confidentiality laws, regulations, and professional practices should address how to minimize the possibility that insurance, pharmacy, and medical records disclose that a person has preclinical AD [124]. From a practical point of view, insurance coverage may provide a challenge, especially in the absence of symptoms. Efforts are ongoing to develop guidelines for the design of studies able to provide robust evidence for insurer coverage determinations [125].

\section{Summary and Conclusions}

The AD field is experiencing an exciting evolution, in both its appreciation of the dynamic nature of the disease and the development of therapeutic strategies aimed at modifying the underlying disease processes (as opposed to simply ameliorating dementia symptoms), and biomarkers have been at the forefront of these paradigm shifts. The core CSF analytes, $\mathrm{A} \beta 42$, tau, and ptau, have withstood the test of time in identifying persons with $\mathrm{AD}$ dementia and have also been shown to strongly predict the development of future cognitive decline in individuals at very early and predementia as well as preclinical stages. In response to these findings, revisions to $\mathrm{AD}$ diagnostic criteria so to incorporate biomarker results have recently been proposed in order to increase the confidence of $\mathrm{AD}$ as the underlying etiology of a clinical impairment and to permit a diagnosis of $\mathrm{AD}$ across the continuum of the disease, eventually perhaps in the asymptomatic period. Although currently proposed for use only in research settings, a diagnosis of preclinical $\mathrm{AD}$ would provide a potential window for therapeutic interventions aimed at preventing cognitive decline not just delaying or halting it once impairments are already clinically apparent. Information regarding biomarker trajectories over the normal course of the disease is being used to inform the design of clinical trials and the evaluation of efficacy of proposed diseasemodifying therapies. Eventually the hope is to bring validated biomarkers to clinical practice in order to provide a more accurate diagnosis, prognostic information regarding future cognitive decline, and evaluation of therapeutic efficacy once disease-modifying treatments become available. Realization of this goal requires worldwide biomarker standardization efforts, consensus among researchers and clinicians regarding the clinical utility of assessing biomarkers in patient care settings, and eventually the endorsement and adoption of such procedures and practices into global health care systems.
Given the large and rapidly expanding aging population, such efforts should be considered a top international priority.

\section{Conflict of Interests}

The author declares that there is no conflict of interests regarding the publication of this paper.

\section{References}

[1] B. D. James, S. E. Leurgans, L. E. Hebert et al., "Contribution of Alzheimer disease to mortality in the United States," Neurology, vol. 82, pp. 1045-1050, 2014.

[2] S. S. Mirra, A. Heyman, D. McKeel et al., "The Consortium to Establish a Registry for Alzheimer's Disease (CERAD). Part II. Standardization of the neuropathologic assessment of Alzheimer's disease," Neurology, vol. 41, no. 4, pp. 479-486, 1991.

[3] Z.S. Khachaturian, "Diagnosis of Alzheimer's disease," Archives of Neurology, vol. 42, no. 11, pp. 1097-1105, 1985.

[4] B. T. Hyman and J. Q. Trojanowski, "Consensus recommendations for the postmortem diagnosis of Alzheimer disease from the National Institute on Aging and the Reagan Institute Working Group on diagnostic criteria for the neuropathological assessment of Alzheimer disease," Journal of neuropathology and experimental neurology, vol. 56, no. 10, pp. 1095-1097, 1997.

[5] T. J. Montine, C. H. Phelps, T. G. Beach et al., "National institute on aging-Alzheimer's association guidelines for the neuropathologic assessment of Alzheimer's disease: a practical approach," Acta Neuropathologica, vol. 123, no. 1, pp. 1-11, 2012.

[6] D. M. Holtzman, J. C. Morris, and A. M. Goate, "Alzheimer's disease: the challenge of the second century," Science Translational Medicine, vol. 3, no. 77, Article ID 77srl, 2011.

[7] G. McKhann, D. Drachman, and M. Folstein, "Clinical diagnosis of Alzheimer's disease: report of the NINCDS-ADRDA work group under the auspices of Department of Health and Human Services Task Force on Alzheimer's disease," Neurology, vol. 34, no. 7, pp. 939-944, 1984.

[8] T. G. Beach, S. E. Monsell, L. E. Phillips, and W. Kukull, "Accuracy of the clinical diagnosis of Alzheimer disease at national institute on aging Alzheimer disease centers, 20052010," Journal of Neuropathology and Experimental Neurology, vol. 71, no. 4, pp. 266-273, 2012.

[9] R. Handels, P. Aalten, C. A. Wolfs et al., "Diagnostic and economic evaluation of new biomarkers for Alzheimers disease: the research protocol of a prospective cohort study," $B M C$ Neurology, vol. 12, p. 72, 2012.

[10] J. L. Cummings and D. V. Jeste, “Alzheimer's disease and its management in the year 2010," Psychiatric Services, vol. 50, no. 9, pp. 1173-1177, 1999.

[11] C. S. Teel, "Rural practitioners' experiences in dementia diagnosis and treatment," Aging and Mental Health, vol. 8, no. 5, pp. 422-429, 2004.

[12] J. Hassenstab, J. Burns, and J. Morris, "Clinical and neuropsychological features of Alzheimer's disease," in Neurobiology of Mental Illness, D. Charney and E. J. Nestler, Eds., pp. 791-804, Oxford University Press, Oxford, UK, 4th edition, 2013.

[13] B. Dubois, H. H. Feldman, C. Jacova et al., "Revising the definition of Alzheimer's disease: a new lexicon," Lancet Neurology, vol. 9, no. 11, pp. 1118-1127, 2010. 
[14] G. M. McKhann, D. S. Knopman, H. Chertkow et al., "The diagnosis of dementia due to Alzheimers disease: Recommendations from the National Institute on Aging and the Alzheimers Association Workgroup," Alzheimer's \& Dementia, vol. 7, pp. 263-269, 2011.

[15] M. Albert, S. T. DeKosky, and D. Dickson, "The diagnosis of Mild Cognitive Impairment due to Alzheimer's disease: recommendations from the National Institute on Aging and Alzheimer's Association Workgroup," Alzheimer's \& Dementia, vol. 7, no. 3, pp. 270-279, 2011.

[16] R. A. Sperling, P. S. Aisen, L. A. Beckett et al., "Toward defining the preclinical stages of Alzheimer's disease: recommendations from the National Institute on Aging-Alzheimer's Association workgroups on diagnostic guidelines for Alzheimer's disease," Alzheimer's \& Dementia, vol. 7, no. 3, pp. 280-292, 2011.

[17] J. Morris, K. Blennow, L. Froelich et al., "Harmonized diagnostic criteria for Alzheimer's disease: recommendations," Journal of Internal Medicine, vol. 275, pp. 204-213, 2014.

[18] K. Blennow, "Biomarkers in Alzheimer's disease drug development," Nature Medicine, vol. 16, no. 11, pp. 1218-1222, 2010.

[19] S. T. Vilming, H. Schrader, and I. Monstad, "The significance of age, sex, and cerebrospinal fluid pressure in post-lumbarpuncture headache," Cephalalgia, vol. 9, no. 2, pp. 99-106, 1989.

[20] S. V. Ahmed, C. Jayawarna, and E. Jude, "Post lumbar puncture headache: diagnosis and management," Postgraduate Medical Journal, vol. 82, no. 973, pp. 713-716, 2006.

[21] K. Blennow, A. Wallin, and O. Häger, "Low frequency of post-lumbar puncture headache in demented patients," Acta Neurologica Scandinavica, vol. 88, no. 3, pp. 221-223, 1993.

[22] E. R. Peskind, R. Riekse, J. F. Quinn et al., "Safety and acceptability of the research lumbar puncture," Alzheimer Disease and Associated Disorders, vol. 19, no. 4, pp. 220-225, 2005.

[23] E. Peskind, A. Nordberg, T. Darreh-Shori, and H. Soininen, "Safety of lumbar puncture procedures in patients with Alzheimer's disease," Current Alzheimer Research, vol. 6, no. 3, pp. 290-292, 2009.

[24] D. Strozyk, K. Blennow, L. R. White, and L. J. Launer, "CSF Abeta 42 levels correlate with amyloid-neuropathology in a population-based autopsy study," Neurology, vol. 60, no. 4, pp. 652-656, 2003.

[25] T. Tapiola, I. Alafuzoff, S. Herukka et al., "Cerebrospinal fluid $\beta$-amyloid 42 and tau proteins as biomarkers of Alzheimer-type pathologic changes in the brain," Archives of Neurology, vol. 66, no. 3, pp. 382-389, 2009.

[26] C. M. Clark, S. Xie, J. Chittams et al., "Cerebrospinal fluid tau and $\beta$-amyloid: how well do these biomarkers reflect autopsyconfirmed dementia diagnoses?" Archives of Neurology, vol. 60, no. 12, pp. 1696-1702, 2003.

[27] A. Fagan, M. A. Mintun, R. H. Mach et al., "Inverse relation between in vivo amyloid imaging load and cerebrospinal fluid A $\beta_{42}$ in humans," Annals of Neurology, vol. 59, no. 3, pp. 512-519, 2006.

[28] A. Forsberg, H. Engler, O. Almkvist et al., "PET imaging of amyloid deposition in patients with mild cognitive impairment," Neurobiology of Aging, vol. 29, no. 10, pp. 1456-1465, 2008.

[29] T. Grimmer, M. Riemenschneider, H. Förstl et al., " Beta amyloid in Alzheimer's disease: Increased deposition in brain is reflected in reduced concentration in cerebrospinal fluid," Biological Psychiatry, vol. 65, no. 11, pp. 927-934, 2009.
[30] N. Tolboom, W. M. van der Flier, M. Yaqub et al., "Relationship of cerebrospinal fluid markers to ${ }^{11} \mathrm{C}-\mathrm{PiB}$ and ${ }^{18} \mathrm{~F}-\mathrm{FDDNP}$ binding," Journal of Nuclear Medicine, vol. 50, no. 9, pp. 1464-1470, 2009.

[31] W. J. Jagust, S. M. Landau, L. M. Shaw et al., "Relationships between biomarkers in aging and dementia," Neurology, vol. 73, no. 15, pp. 1193-1199, 2009.

[32] S. Hong, O. Quintero-Monzon, B. L. Ostaszewski et al., "Dynamic analysis of amyloid $\beta$-protein in behaving mice reveals opposing changes in isf versus parenchymal $\mathrm{A} \beta$ during age-related plaque formation," Journal of Neuroscience, vol. 31, no. 44, pp. 15861-15869, 2011.

[33] A. M. Fagan, M. A. Mintun, A. R. Shah et al., "Cerebrospinal fluid tau and $\mathrm{ptau}_{181}$ increase with cortical amyloid deposition in cognitively normal individuals: Implications for future clinical trials of Alzheimer's disease," EMBO Molecular Medicine, vol. 1, no. 8-9, pp. 371-380, 2009.

[34] D. Irwin, J. Trojanowski, and M. Grossman, "Cerebrospinal fluid biomarkers for differentiation of frontotemporal lobar degeneration from Alzheimer's disease," Frontiers in Aging Neuroscience, vol. 5, no. 6, 2013.

[35] C. Yao, A. J. Williams, A. K. Ottens et al., "Detection of protein biomarkers using high-throughput immunoblotting following focal ischemic or penetrating ballistic-like brain injuries in rats," Brain Injury, vol. 22, no. 10, pp. 723-732, 2008.

[36] M. Öst, K. Nylén, L. Csajbok et al., "Initial CSF total tau correlates with 1-year outcome in patients with traumatic brain injury," Neurology, vol. 67, no. 9, pp. 1600-1604, 2006.

[37] K. Blennow, "Cerebrospinal fluid protein biomarkers for Alzheimer's disease," NeuroRx, vol. 1, no. 2, pp. 213-225, 2004.

[38] M. Otto, J. Wiltfang, H. Tumani et al., "Elevated levels of tauprotein in cerebrospinal fluid of patients with Creutzfeldt-Jakob disease," Neuroscience Letters, vol. 225, no. 3, pp. 210-212, 1997.

[39] M. B. Coulthart, G. H. Jansen, E. Olsen et al., "Diagnostic accuracy of cerebrospinal fluid protein markers for sporadic Creutzfeldt-Jakob disease in Canada: a 6-year prospective study," BMC Neurology, vol. 11, article 133, 2011.

[40] T. Skillbäck, C. Rosén, F. Asztely, N. Mattsson, K. Blennow, and H. Zetterberg, "Diagnostic performance of cerebrospinal fluid total tau and phosphorylated tau in Creutzfeldt-Jakob disease: results from the Swedish Mortality Registry," JAMA Neurology, vol. 71, no. 4, pp. 476-483, 2014.

[41] N. Kfoury, B. B. Holmes, H. Jiang, D. M. Holtzman, and M. I. Diamond, "Trans-cellular propagation of Tau aggregation by fibrillar species," The Journal of Biological Chemistry, vol. 287, no. 23, pp. 19440-19451, 2012.

[42] K. Yamada, J. R. Cirrito, F. R. Stewart et al., "In vivo microdialysis reveals age-dependent decrease of brain interstitial fluid tau levels in P301S human tau transgenic mice," Journal of Neuroscience, vol. 31, no. 37, pp. 13110-13117, 2011.

[43] K. Yamada, J. K. Holth, F. Liao et al., "Neuronal activity regulates extracellular tau in vivo," The Journal of Experimental Medicine, vol. 211, no. 3, pp. 387-393, 2014.

[44] H. Hampel, K. Blennow, L. M. Shaw, Y. C. Hoessler, H. Zetterberg, and J. Q. Trojanowski, "Total and phosphorylated tau protein as biological markers of Alzheimer's disease," Experimental Gerontology, vol. 45, no. 1, pp. 30-40, 2010.

[45] K. Buerger, M. Ewers, T. Pirttilä et al., "CSF phosphorylated tau protein correlates with neocortical neurofibrillary pathology in Alzheimer's disease," Brain, vol. 129, no. 11, pp. 3035-3041, 2006. 
[46] D. Chien, A. K. Szardenings, S. Bahri et al., "Early clinical PET imaging results with the novel PHF-tau radioligand [F18]T808," Journal of Alzheimer's Disease, vol. 38, no. 1, pp. 171-184, 2014.

[47] A. M. Fagan and R. J. Perrin, "Upcoming candidate cerebrospinal fluid biomarkers of Alzheimer's disease," Biomarkers in Medicine, vol. 6, no. 4, pp. 455-476, 2012.

[48] J. Lee, K. Blennow, N. Andreasen et al., "The brain injury biomarker VLP-1 is increased in the cerebrospinal fluid of Alzheimer disease patients," Clinical Chemistry, vol. 54, no. 10, pp. 1617-1623, 2008.

[49] R. Craig-Schapiro, R. J. Perrin, C. M. Roe et al., "YKL-40: a novel prognostic fluid biomarker for preclinical Alzheimer's disease," Biological Psychiatry, vol. 68, pp. 903-912, 2010.

[50] R. Tarawneh, G. D’Angelo, E. MacY et al., "Visinin-like protein1: diagnostic and prognostic biomarker in Alzheimer disease," Annals of Neurology, vol. 70, no. 2, pp. 274-285, 2011.

[51] R. J. Perrin, R. Craig-Schapiro, J. P. Malone et al., "Identification and validation of novel cerebrospinal fluid biomarkers for staging early Alzheimer's disease," PLoS ONE, vol. 6, no. 1, Article ID e16032, 2011.

[52] B. Olsson, J. Hertze, R. Lautner et al., "Microglial markers are elevated in the prodromal phase of Alzheimer's disease and vascular dementia," Journal of Alzheimer's Disease, vol. 33, no. 1, pp. 45-53, 2013.

[53] X. Luo, L. Hou, H. Shi et al., "CSF levels of the neuronal injury biomarker visinin-like protein-1 in alzheimer's disease and dementia with lewy bodies," Journal of Neurochemistry, vol. 127, no. 5, pp. 681-690, 2013.

[54] R. Tarawneh, J.-M. Lee, J. H. Ladenson, J. C. Morris, and D. M. Holtzman, "CSF VILIP-1 predicts rates of cognitive decline in early Alzheimer disease," Neurology, vol. 78, no. 10, pp. 709-719, 2012.

[55] K. Blennow, H. Hampel, M. Weiner, and H. Zetterberg, "Cerebrospinal fluid and plasma biomarkers in Alzheimer disease," Nature Reviews Neurology, vol. 6, no. 3, pp. 131-144, 2010.

[56] C. M. Hulette, K. A. Welsh-Bohmer, M. G. Murray, A. M. Saunders, D. C. Mash, and L. M. McIntyre, "Neuropathological and neurolasychological changes in "normal" aging: evidence for preclinical Alzheimer Disease in cognitively normal individuals," Journal of Neuropathology and Experimental Neurology, vol. 57, no. 12, pp. 1168-1174, 1998.

[57] J. L. Price and J. C. Morris, "Tangles and plaques in nondemented aging and "preclinical" Alzheimer's disease," Annals of Neurology, vol. 45, pp. 358-368, 1999.

[58] D. Galasko, L. Chang, R. Motter et al., "High cerebrospinal fluid tau and low amyloid $\beta 42$ levels in the clinical diagnosis of Alzheimer 's disease and relation to apolipoprotein E genotype," Archives of Neurology, vol. 55, no. 7, pp. 937-945, 1998.

[59] A. Maddalena, A. Papassotiropoulos, B. Müller-Tillmanns et al., "Biochemical diagnosis of Alzheimer disease by measuring the cerebrospinal fluid ratio of phosphorylated tau protein to $\beta$ amyloid peptide42," Archives of Neurology, vol. 60, no. 9, pp. 1202-1206, 2003.

[60] G. M. Halliday, J. L. Holton, T. Revesz, and D. W. Dickson, "Neuropathology underlying clinical variability in patients with synucleinopathies," Acta Neuropathologica, vol. 122, no. 2, pp. 187-204, 2011.

[61] N. S. M. Schoonenboom, F. E. Reesink, N. A. Verwey et al., "Cerebrospinal fluid markers for differential dementia diagnosis in a large memory clinic cohort," Neurology, vol. 78, no. 1, pp. 47-54, 2012.
[62] R. C. Petersen, G. E. Smith, S. C. Waring, R. J. Ivnik, E. G. Tangalos, and E. Kokmen, "Mild cognitive impairment: clinical characterization and outcome," Archives of Neurology, vol. 56, pp. 303-308, 1999.

[63] R. C. Petersen, "Mild cognitive impairment as a diagnostic entity," Journal of Internal Medicine, vol. 256, no. 3, pp. 183-194, 2004.

[64] M. Weiner, D. P. Veitch, P. S. Aisen et al., “The Alzheimer's disease neuroimaging initiative: a review of papers published since its inception," Alzheimer's \& Dementia, vol. 9, no. 5, pp. el11-e194, 2013.

[65] L. M. Shaw, H. Vanderstichele, M. Knapik-Czajka et al., "Cerebrospinal fluid biomarker signature in alzheimer's disease neuroimaging initiative subjects," Annals of Neurology, vol. 65, no. 4, pp. 403-413, 2009.

[66] C. C. Rowe, S. Ng, U. Ackermann et al., "Imaging $\beta$-amyloid burden in aging and dementia," Neurology, vol. 68, no. 20, pp. 1718-1725, 2007.

[67] N. Mattsson, H. Zetterberg, O. Hansson et al., "CSF biomarkers and incipient Alzheimer disease in patients with mild cognitive impairment," Journal of the American Medical Association, vol. 302, no. 4, pp. 385-393, 2009.

[68] P. J. Visser, F. Verhey, D. L. Knol et al., "Prevalence and prognostic value of CSF markers of Alzheimer's disease pathology in patients with subjective cognitive impairment or mild cognitive impairment in the DESCRIPA study: a prospective cohort study," The Lancet Neurology, vol. 8, no. 7, pp. 619-627, 2009.

[69] P. Buchhave, L. Minthon, H. Zetterberg, A. K. Wallin, K. Blennow, and O. Hansson, "Cerebrospinal fluid levels of $\beta$ amyloid 1-42, but not of tau, are fully changed already 5 to 10 years before the onset of Alzheimer dementia," Archives of General Psychiatry, vol. 69, no. 1, pp. 98-106, 2012.

[70] B. J. Snider, A. M. Fagan, C. Roe et al., "Cerebrospinal fluid biomarkers and rate of cognitive decline in very mild dementia of the Alzheimer type," Archives of Neurology, vol. 66, no. 5, pp. 638-645, 2009.

[71] H. Braak and E. Braak, "Frequency of stages of Alzheimerrelated lesions in different age categories," Neurobiology of Aging, vol. 18, no. 4, pp. 351-357, 1997.

[72] J. L. Price, D. W. McKeel Jr., V. D. Buckles et al., "Neuropathology of nondemented aging: presumptive evidence for preclinical Alzheimer disease," Neurobiology of Aging, vol. 30, no. 7, pp. 1026-1036, 2009.

[73] S. Vos, C. Xiong, P. J. Visser et al., "Preclinical Alzheimer's disease and its outcome: a longitudinal cohort study," The Lancet Neurology, vol. 12, no. 10, pp. 957-965, 2013.

[74] C. R. Jack Jr., D. S. Knopman, S. D. Weigand et al., "An operational approach to National Institute on Aging-Alzheimer's Association criteria for preclinical Alzheimer disease," Annals of Neurology, vol. 71, no. 6, pp. 765-775, 2012.

[75] I. Skoog, P. Davidsson, Ó. Aevarsson, H. Vanderstichele, E. Vanmechelen, and K. Blennow, "Cerebrospinal fluid betaamyloid 42 is reduced before the onset of sporadic dementia: a population-based study in 85-year-olds," Dementia and Geriatric Cognitive Disorders, vol. 15, no. 3, pp. 169-176, 2003.

[76] D. R. Gustafson, I. Skoog, L. Rosengren, H. Zetterberg, and K. Blennow, "Cerebrospinal fluid $\beta$-amyloid 1-42 concentration may predict cognitive decline in older women," Journal of Neurology, Neurosurgery and Psychiatry, vol. 78, no. 5, pp. 461464, 2007.

[77] E. Stomrud, O. Hansson, K. Blennow, L. Minthon, and E. Londos, "Cerebrospinal fluid biomarkers predict decline in 
subjective cognitive function over 3 years in healthy elderly," Dementia and Geriatric Cognitive Disorders, vol. 24, no. 2, pp. 118-124, 2007.

[78] A. M. Fagan, C. M. Roe, C. Xiong, M. A. Mintun, J. C. Morris, and D. M. Holtzman, "Cerebrospinal fluid tau/ $\beta$-amyloid 42 ratio as a prediction of cognitive decline in nondemented older adults," Archives of Neurology, vol. 64, no. 3, pp. 343-349, 2007.

[79] G. Li, I. Sokal, J. F. Quinn et al., "CSF tau/A $\beta_{42}$ ratio for increased risk of mild cognitive impairment: a follow-up study," Neurology, vol. 69, no. 7, pp. 631-639, 2007.

[80] O. Hansson, H. Zetterberg, P. Buchhave, E. Londos, K. Blennow, and L. Minthon, "Association between CSF biomarkers and incipient Alzheimer's disease in patients with mild cognitive impairment: a follow-up study," The Lancet Neurology, vol. 5, no. 3, pp. 228-234, 2006.

[81] K. G. Mawuenyega, W. Sigurdson, V. Ovod et al., "Decreased clearance of CNS $\beta$-amyloid in Alzheimer's disease," Science, vol. 330, no. 6012, p. 1774, 2010.

[82] R. J. Bateman, P. S. Aisen, B. de Strooper et al., "Autosomaldominant Alzheimer's disease: a review and proposal for the prevention of Alzheimer's disease," Alzheimer's Research and Therapy, vol. 2, no. 6, article 35, 2011.

[83] M. Moonis, J. M. Swearer, M. P. E. Dayaw et al., "Familial Alzheimer disease: decreases in CSF A $\beta 42$ levels precede cognitive decline," Neurology, vol. 65, no. 2, pp. 323-325, 2005.

[84] R. Bateman, C. Xiong, T. L. S. Benzinger et al., "Clinical and biomarker changes in dominantly inherited Alzheimer's disease," The New England Journal of Medicine, vol. 367, pp. 795804, 2012.

[85] A. Fagan, "Longitudinal change in CSF biomarkers in autosomal-dominant Alzheimer disease," Science Translational Medicine, vol. 6, no. 226, p. 226ra30, 2014.

[86] T. Tapiola, H. Soininen, and T. Pirttilä, "CSF tau and A $\beta 42$ levels in patients with Down's syndrome," Neurology, vol. 56, no. 7, pp. 979-980, 2001.

[87] J. M. Ringman, S. G. Younkin, D. Pratico et al., "Biochemical markers in persons with preclinical familial Alzheimer disease," Neurology, vol. 71, no. 2, pp. 85-92, 2008.

[88] C. R. Jack, D. S. Knopman, W. J. Jagust et al., “Tracking pathophysiological processes in Alzheimer's disease: an updated hypothetical model of dynamic biomarkers," The Lancet Neurology, vol. 12, no. 2, pp. 207-216, 2013.

[89] R. J. Perrin, A. M. Fagan, and D. M. Holtzman, "Multimodal techniques for diagnosis and prognosis of Alzheimer's disease," Nature, vol. 461, no. 7266, pp. 916-922, 2009.

[90] C. R. Jack Jr., D. S. Knopman, W. J. Jagust et al., "Hypothetical model of dynamic biomarkers of the Alzheimer's pathological cascade," The Lancet Neurology, vol. 9, no. 1, pp. 119-128, 2010.

[91] R. S. Desikan, L. K. McEvoy, W. K. Thompson et al., "Amyloid$\beta$-associated clinical decline occurs only in the presence of elevated P-tau.," Archives of Neurology, vol. 69, no. 6, pp. 709713, 2012.

[92] M. I. Kester, A. E. Van Der Vlies, M. A. Blankenstein et al., "CSF biomarkers predict rate of cognitive decline in Alzheimer disease," Neurology, vol. 73, no. 17, pp. 1353-1358, 2009.

[93] I. A. van Rossum, S. J. B. Vos, L. Burns et al., "Injury markers predict time to dementia in subjects with MCI and amyloid pathology," Neurology, vol. 79, no. 17, pp. 1809-1816, 2012.

[94] K. Blennow, H. Zetterberg, L. Minthon et al., "Longitudinal stability of CSF biomarkers in Alzheimer's disease," Neuroscience Letters, vol. 419, no. 1, pp. 18-22, 2007.
[95] H. Zetterberg, M. Pedersen, K. Lind et al., "Intra-individual stability of CSF biomarkers for Alzheimer's disease over two years," Journal of Alzheimer's Disease, vol. 12, no. 3, pp. 255-260, 2007.

[96] L. A. Beckett, D. J. Harvey, A. Gamst et al., "The Alzheimer's Disease neuroimaging initiative: annual change in biomarkers and clinical outcomes," Alzheimer's and Dementia, vol. 6, no. 3, pp. 257-264, 2010.

[97] P. Vemuri, H. J. Wiste, S. D. Weigand et al., "Serial MRI and CSF biomarkers in normal aging, MCI, and AD," Neurology, vol. 75, no. 2, pp. 143-151, 2010.

[98] M. Kanai, E. Matsubara, K. Isoe et al., "Longitudinal study of cerebrospinal fluid levels of tau, A betal-40, and A betal-42(43) in Alzheimer's disease: a study in Japan," Annals of Neurology, vol. 44, pp. 17-26, 1998.

[99] T. Sunderland, B. Wolozin, D. Galasko et al., "Longitudinal stability of CSF tau levels in Alzheimer patients," Biological Psychiatry, vol. 46, no. 6, pp. 750-755, 1999.

[100] J. B. Toledo, S. X. Xie, J. Q. Trojanowski, and L. M. Shaw, "Longitudinal change in CSF Tau and A $\beta$ biomarkers for up to 48 months in ADNI," Acta Neuropathologica, vol. 126, pp. 659670, 2013.

[101] C. R. Jack, M. S. Albert, D. S. Knopman et al., "Introduction to the recommendations from the National Institute on AgingAlzheimer's Association workgroups on diagnostic guidelines for Alzheimer's disease," Alzheimer's \& Dementia, vol. 7, no. 3, pp. 257-262, 2011.

[102] M. I. Kester, L. Boelaarts, F. H. Bouwman et al., "Diagnostic impact of CSF biomarkers in a local hospital memory clinic," Dementia and Geriatric Cognitive Disorders, vol. 29, no. 6, pp. 491-497, 2010.

[103] F. Mouton-Liger, D. Wallon, A. C. Troussière et al., "Impact of cerebro-spinal fluid biomarkers of Alzheimer's disease in clinical practice: a multicentric study," Journal of Neurology, vol. 261, no. 1, pp. 144-151, 2014.

[104] A. Troussière, D. Wallon, F. Mouton-Liger et al., "Who needs cerebrospinal biomarkers? A national survey in clinical practice," Journal of Alzheimer's Disease, vol. 40, pp. 857-861, 2014.

[105] D. S. Knopman, C. R. Jack Jr., H. J. Wiste et al., "Short-term clinical outcomes for stages of NIA-AA preclinical Alzheimer disease," Neurology, vol. 78, no. 20, pp. 1576-1582, 2012.

[106] J. C. Morris and D. J. Selkoe, "Recommendations for the incorporation of biomarkers into Alzheimer clinical trials: an overview," Neurobiology of Aging, vol. 32, supplement 1, pp. S1S3, 2011.

[107] R. A. Sperling, C. R. Jack Jr., and P. S. Aisen, "Testing the right target and right drug at the right stage," Science Translational Medicine, vol. 3, no. 111, Article ID 111cm33, 2011.

[108] J. Langbaum, A. S. Fleisher, K. Chen et al., "Ushering in the study and treatment of preclinical Alzheimer disease," Nature Reviews Neurology, vol. 9, no. 7, pp. 371-381, 2013.

[109] R. Sperling, D. M. Rentz, K. A. Johnson et al., “The A4 study: stopping AD before symptoms begin?" Science Translational Medicine, vol. 6, no. 228, p. 228fs13, 2014.

[110] E. M. Reiman, J. B. S. Langbaum, A. S. Fleisher et al., "Alzheimers prevention initiative: a plan to accelerate the evaluation of presymptomatic treatments," Journal of Alzheimer's Disease, vol. 26, supplement 3, no. 3, pp. 321-329, 2011.

[111] S. Mills, J. Mallmann, A. M. Santacruz et al., "Preclinical trials in autosomal dominant AD: implementation of the DIAN-TU trial," Revue Neurologique, vol. 169, no. 10, pp. 737-743, 2013. 
[112] N. Mattsson, U. Andreasson, S. Persson et al., “The Alzheimer's Association external quality control program for cerebrospinal fluid biomarkers," Alzheimer's and Dementia, vol. 7, no. 4, pp. 386.e6-395.e6, 2011.

[113] N. Mattsson, U. Andreasson, S. Persson et al., "CSF biomarker variability in the Alzheimer's association quality control program," Alzheimer's and Dementia, vol. 9, no. 3, pp. 251-261, 2013.

[114] H. M. Vanderstichele, L. Shaw, M. Vandijck et al., "Alzheimer disease biomarker testing in cerebrospinal fluid: a method to harmonize assay platforms in the absence of an absolute reference standard," Clinical Chemistry, vol. 59, no. 4, pp. 710$712,2013$.

[115] M. T. Fodero-Tavoletti, S. Furumoto, L. Taylor et al., "Assessing THK523 selectivity for tau deposits in Alzheimer's disease and non Alzheimer's disease tauopathies," Alzheimer's Research \& Therapy, vol. 6, no. 1, p. 11, 2014.

[116] P. Giannetti, M. Politis, P. Su et al., "Microglia activation in multiple sclerosis black holes predicts outcome in progressive patients: an in vivo [(11)C](R)-PK11195-PET pilot study," Neurobiology of Disease, vol. 65, pp. 203-210, 2014.

[117] J. W. Bartlett, C. Frost, N. Mattsson et al., "Determining cutpoints for Alzheimer's disease biomarkers: statistical issues, methods and challenges," Biomarkers in Medicine, vol. 6, no. 4, pp. 391-400, 2012.

[118] J. Luo and C. Xiong, "Youden index and associated cutpoints for three ordinal diagnostic groups," Communications in Statistics. Simulation and Computation, vol. 42, no. 6, pp. 12131234, 2013.

[119] S. Vos, I. van Rossum, L. Burns et al., "Test sequence of CSF and MRI biomarkers for prediction of AD in subjects with MCI," Neurobiology of Aging, vol. 33, no. 10, pp. 2272-2281, 2012.

[120] C. Xiong, G. van Belle, K. Chen et al., "Combining multiple markers to improve the longitudinal rate of progressionapplication to clinical trials on the early stage of Alzheimer's disease," Statistics in Biopharmaceutical Research, vol. 5, no. 1, 2013.

[121] C. Bazenet and S. Lovestone, "Plasma biomarkers for Alzheimers disease: much needed but tough to find," Biomarkers in Medicine, vol. 6, no. 4, pp. 441-454, 2012.

[122] D. Alcolea, P. Martínez-Lage, A. Izagirre et al., "Feasibility of lumbar puncture in the study of cerebrospinal fluid biomarkers for Alzheimer's disease: a multicenter study in Spain," Journal of Alzheimer's Disease, vol. 39, pp. 719-726, 2014.

[123] H. Vanderstichele, M. Bibl, S. Engelborghs et al., "Standardization of preanalytical aspects of cerebrospinal fluid biomarker testing for Alzheimer's disease diagnosis: a consensus paper from the Alzheimer's Biomarkers Standardization Initiative," Alzheimer's and Dementia, vol. 8, no. 1, pp. 65-73, 2012.

[124] J. Karlawish, "Addressing the ethical, policy, and social challenges of preclinical Alzheimer disease," Neurology, vol. 77, no. 15, pp. 1487-1493, 2011.

[125] S. Pearson, D. A. Ollendorf, J. A. Colby et al., "Biomarker tests for the diagnosis of Alzheimer's disease: generating evidence to inform insurance coverage determinations," Alzheimer's \& Dementia, vol. 9, no. 7, pp. 745-752, 2013. 


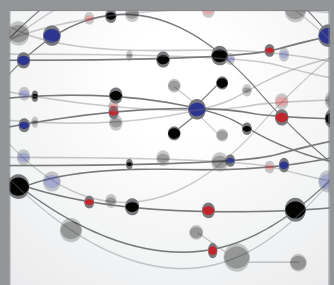

The Scientific World Journal


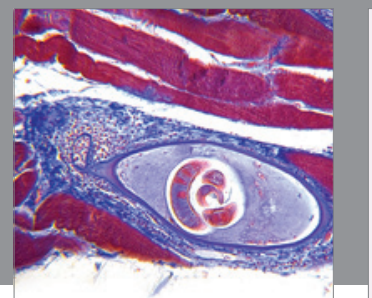

Gastroenterology

Research and Practice


\section{Hindawi}

Submit your manuscripts at

http://www.hindawi.com
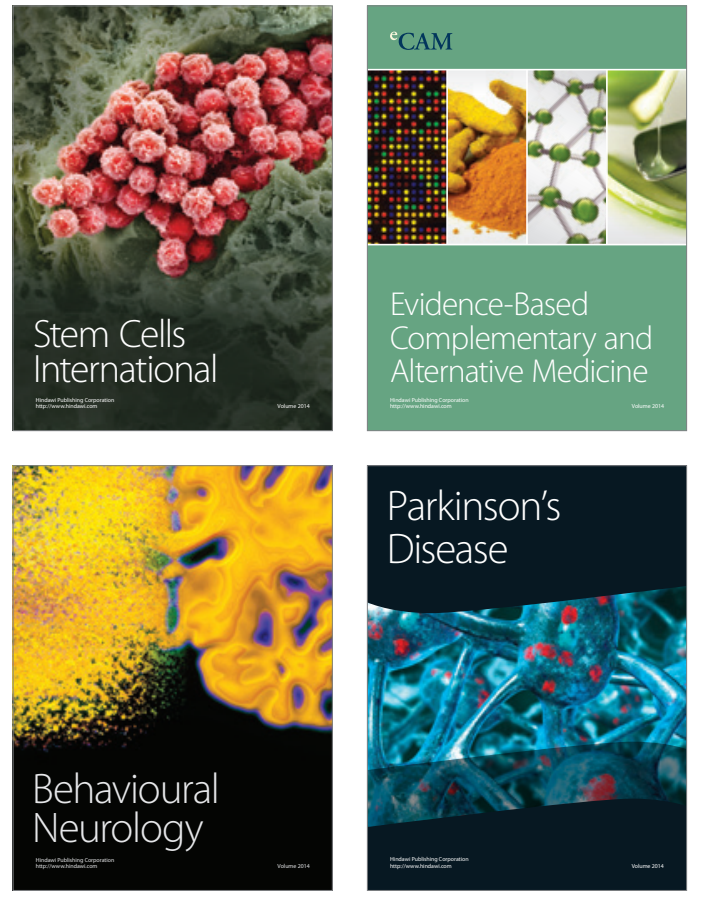


Disease Markers
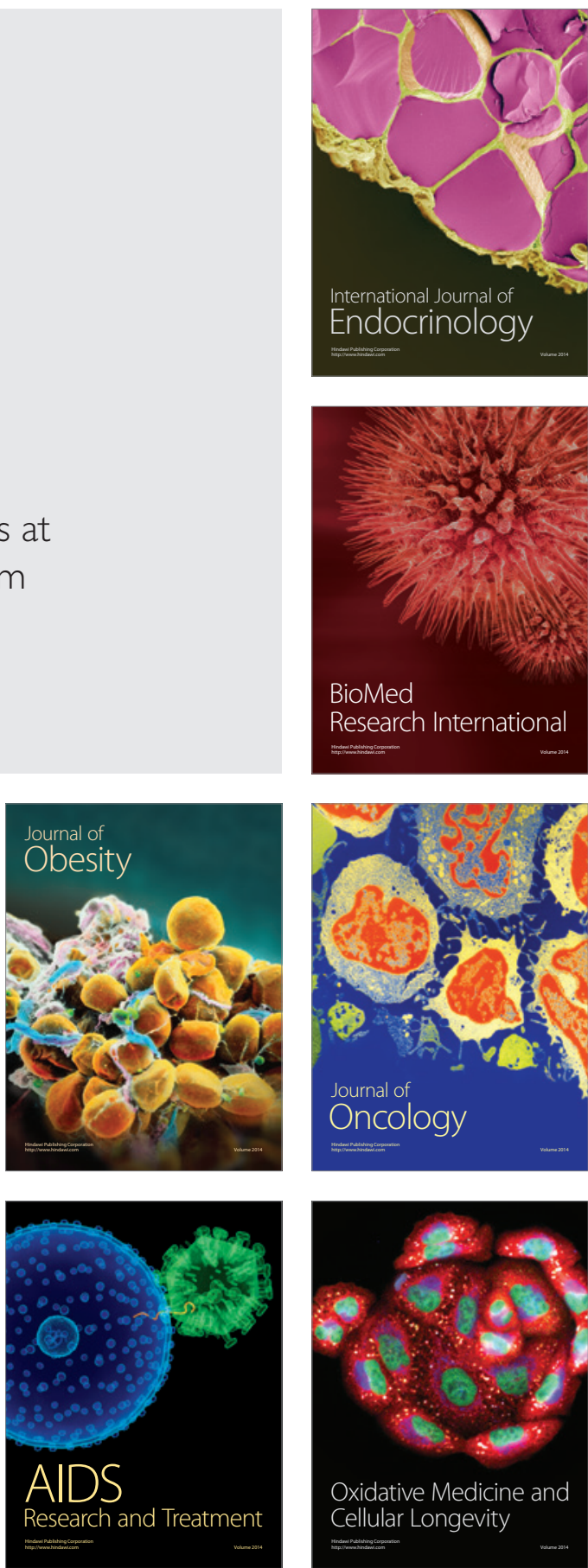